\title{
Influence of seismic activity on the atmospheric electric field in Lisbon (Portugal) from 1955 to 1991
}

\author{
Hugo Gonçalves Silva ${ }^{1, \star}$, Maria Manuela Oliveira ${ }^{2}$, Cláudia Serrano ${ }^{1}$, Mourad Bezzeghoud ${ }^{1}$, \\ António Heitor Reis ${ }^{1}$, Rui Namorado Rosa ${ }^{1}$, Pier Francesco Biagi ${ }^{3}$
}

\author{
${ }^{1}$ Universidade de Évora, Escola de Ciências e Tecnologia, Centro de Geofisica de Évora e Departamento de Física, Évora, Portugal \\ ${ }^{2}$ Universidade de Évora, Escola de Ciências e Tecnologia, Departamento de Matemática, Évora, Portugal \\ ${ }^{3}$ Università di Bari, Dipartimento di Fisica e Centro Interdipartimentale di Ricerca per la Valutazione e Mitigazione del Rischio Sismico e \\ Vulcanico, Bari, Italy
}

Article history

Received August 9, 2011; accepted November 10, 2011.

Subject classification:

Processes and dynamics, Earthquake faults: properties and evolution, Seismic risk, Atmospheric electric field, LAI coupling.

\section{ABSTRACT}

In the present study, we considered the influence of seismic activity on the atmospheric electric field recorded at Portela meteorological station (Lisbon, Portugal) for the period from 1955 to 1991. To this end, an exploratory method was developed, which involved the selection of events for which the distance from the atmospheric electrical field sensor to the earthquake epicenter is smaller than the preparation radius of the event. This enabled the correlation of the atmospheric electric field variations with a quantity $S$, defined basically as the ratio of the earthquake preparation radius to the distance between the sensor and the event epicenter. The first results show promising perspectives, but clearly a more profound study is required, in which a careful analysis of the weather conditions and other variables, like atmospheric radon levels, must be considered.

\section{Introduction}

Since an important article by Pierce in the 1970's [Pierce 1976], many models have been developed to explore the coupling between the lithosphere-atmosphere-ionosphere during seismic events [e.g. Liperovsky et al. 2008, Omori et al. 2009, Harrison et al. 2010, Kachakhidze et al. 2011]. All of these appear to agree that variations in the vertical component of the atmospheric electric field (Ez) are related to the amount of radon that emanates during earthquake preparation stages, which then promotes air ionization and reduces Ez. From hereafter, we adopt the use of the atmospheric electric potential gradient $(\mathrm{PG})$, as defined as: $\mathrm{PG}=-\mathrm{Ez}$.

Moreover, PG anomalies are not only candidates for seismic electromagnetic precursors in the context of shortterm earthquake prediction [Harrison et al. 2010], as they also give important insights into the physics of these phenomena [Kachakhidze et al. 2011]. Nevertheless, there has been little observational work carried out on this subject [e.g. Smirnov 2008, Kachakhize et al. 2009, Silva et al. 2011).

In the present study, we present a new approach for the investigation of the effects of earthquakes on the PG, through the analysis of a dataset recorded at Portela meteorological station (Lisbon, Portugal) for the period from 1955 to 1991 . It is important to note that these data correspond to the vertical and very-slow-varying component of the atmospheric electric field, and therefore the associated magnetic effects are negligible. Thus the phenomenology presented here is completely different from the geomagnetic precursors reported in the literature [e.g. Fraser-Smith et al. 1990]. Moreover, it is also of fundamental importance to note that the variations on the 'atmospheric' electric fields discussed in this study should not be confused with the distinct phenomenon known as seismic electric signals, which are said to be developed in the 'lithosphere' before significant earthquakes, and that have been extensively reported in the literature [e.g. Varotsos and Alexopoulos 1984]. Indeed, for the moment, no direct relationships between the atmospheric electric field variations reported here and both geomagnetic field anomalies and seismoelectric signals in the literature have been shown, and it is beyond the scope of the present study to explore this issue. Additionally, we must emphasize that this is an initial study; nevertheless, interesting trends are found that show that the effects of seismic activity on the PGs are local, and that they tend to reduce the PGs.

The organization of this report is as follows: some considerations relating to the datasets are given in Section 2; the methodology is presented in Section 3; the results are discussed in Section 4; and at the end we present some final remarks, in Section 5. 


\section{The datasets}

In the present study, we considered the hourly PG values recorded at Portela meteorological station (Lisbon, Portugal; $38^{\circ} 47^{\prime} \mathrm{N}, 9^{\circ} 08^{\prime} \mathrm{W}$ ) for the period from 1955 to 1991 . The Portuguese Meteorological Institute recorded these data with a Benndorff electrograph with a 1-m-heigh probe. The data series was interrupted from 1975 to 1977 when the electrometer was switched off for maintenance. The annual behavior of the PG is presented in Figure 1a, where an anomaly associated with the nuclear tests in the 1960's can be seen, together with the slow recover of the PG in the following years. This effect was previously reported in a seminal study [Pierce 1972], and is clearly shown here. The average behavior of the diurnal cycle is also presented in Figure 1b. A global analysis of the different influences on the PG was reported by Serrano et al. [2006], where more details of the dataset can be found.

On the other hand, two seismic catalogs were used in the present study: for the period from 1955 to 1961, we used
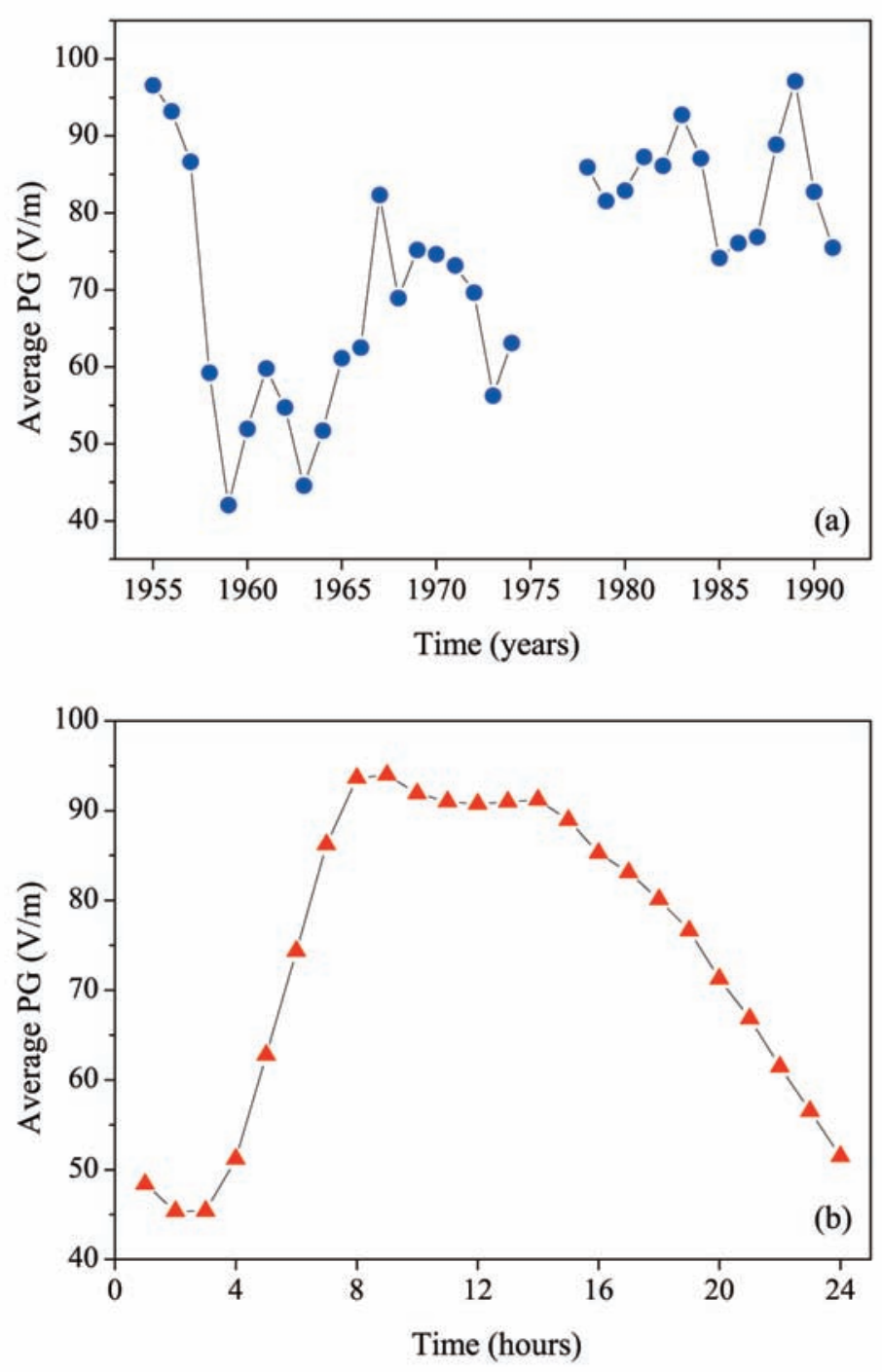

Figure 1. a) Average PGs per year during the period from 1955 to 1991 at Portela (Lisbon, Portugal). b) The diurnal cycle of the PGs averaged over all of the data.

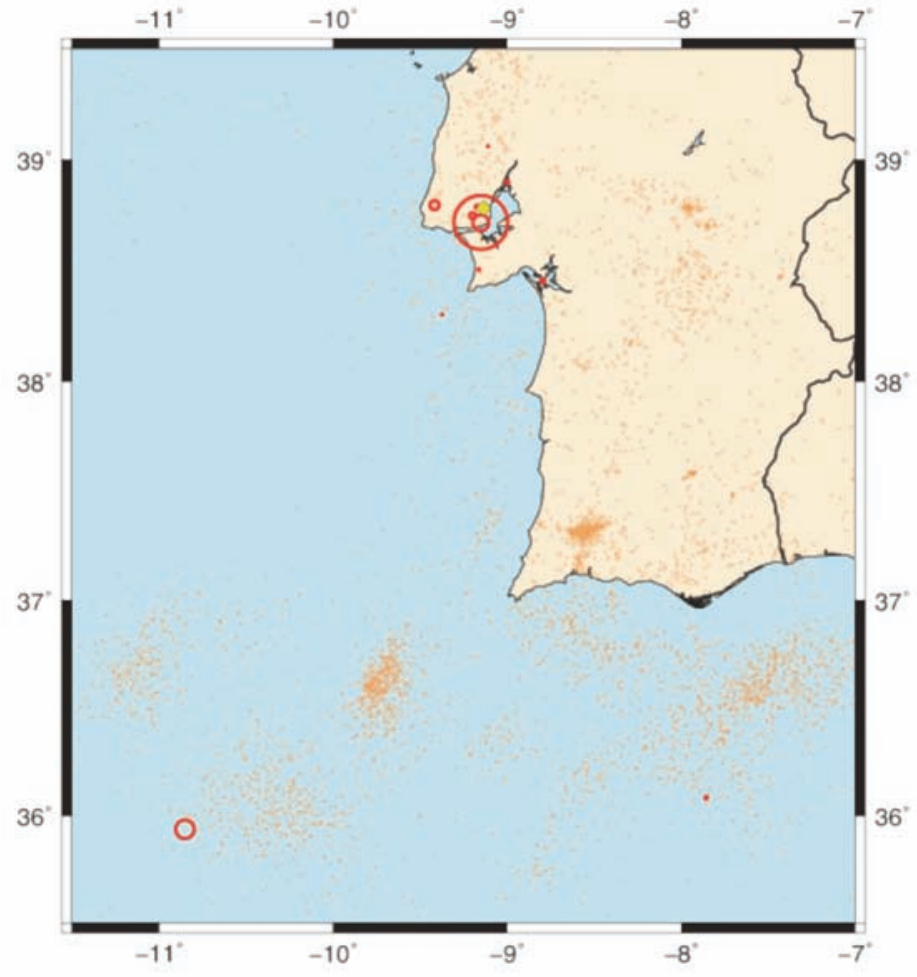

Figure 2. Map of Portugal showing the seismicity (small orange circles) of the south of Portugal for the period from January 1, 1961 to December 31, 2010 (data provided by Portuguese Meteorological Institute, Portugal). The red circles are proportional to $S$ and highlight the most relevant earthquakes.

the catalog from the Portuguese National Laboratory for Civil Engineering [Sousa et al. 1992]; and for the period from 1961 to 1991, we used the catalog from the Portuguese Meteorological Institute (the Portuguese Meteorological Institute database). Figure 2 shows the seismic activity from 1961 to 2010 in the region where the PG sensor was installed. Indeed, the seismicity of this zone has been widely studied mainly because of the well-known Lisbon earthquake of 1755 , and the interested reader can find a concise studies of this earthquake in the literature [i.e. Borges et al. 2001, Buforn et al. 2004].

\section{The methodology}

First of all, it should be noted that the measurements presented in this study are local, in the sense that any perturbation in the PG values must be caused by a disturbance that occurs near to the sensor, as for example, for artificial radioactivity variations [see Serrano et al. 2006]. For this reason, it is highly unexpected that seismic events far away from the sensor will directly affect the PG. In this way, it is necessary to establish a criterion to select the seismic events that might actually influence the PG from those that cannot. At the same time, it is expected that seismic events of greater magnitude do influence the PG at larger distances from the sensor, as compared with smaller seismic events. 


\begin{tabular}{|c|c|c|c|c|c|c|}
\hline $\begin{array}{c}\text { Date } \\
\text { (dd/mm/yyyy) }\end{array}$ & $\begin{array}{l}\text { Time } \\
\text { (days) }\end{array}$ & $\begin{array}{l}\text { Depth } \\
(\mathrm{km})\end{array}$ & $\begin{array}{c}\mathrm{M} \\
(\mathrm{km})\end{array}$ & $\begin{array}{c}\mathrm{R} \\
(\mathrm{km})\end{array}$ & $\mathrm{D}$ & $S$ \\
\hline $17 / 06 / 1955$ & 0.050 & - & 3.4 & 28.973 & 18.138 & 0.597 \\
\hline $30 / 07 / 1959$ & 0.001 & 13 & 4.3 & 70.632 & 5.991 & 10.789 \\
\hline $08 / 05 / 1962$ & 0.625 & - & 3.3 & 26.242 & 6.309 & - \\
\hline $15 / 03 / 1964$ & 0.938 & 10 & 6.2 & 463.447 & 319.056 & 0.453 \\
\hline $31 / 03 / 1964$ & 0.624 & - & 2.7 & 14.488 & 6.633 & 1.184 \\
\hline $26 / 08 / 1966$ & 0.248 & 10 & 4.6 & 95.060 & 87.702 & 0.084 \\
\hline $24 / 02 / 1967$ & 0.926 & 2 & 4.3 & 70.632 & 65.619 & 0.076 \\
\hline $04 / 10 / 1967$ & 0.104 & 13 & 4.3 & 70.632 & 56.341 & 0.254 \\
\hline $24 / 02 / 1969$ & 0.511 & - & 4.5 & 86.099 & 45.420 & 0.896 \\
\hline $28 / 02 / 1969$ & 0.111 & 10 & 7.5 & 1678.804 & 349.917 & 3.798 \\
\hline $14 / 01 / 1973$ & 0.853 & 2 & 4.3 & 70.632 & 25.085 & 1.816 \\
\hline $29 / 06 / 1973$ & 0.665 & 21 & 3.7 & 38.994 & 29.650 & 0.315 \\
\hline $26 / 05 / 1975$ & 0.383 & 10 & 8.1 & 3040.885 & 828.839 & - \\
\hline $09 / 01 / 1987$ & 0.025 & 9 & 2 & 7.244 & 4.311 & 0.680 \\
\hline $22 / 05 / 1988$ & 0.583 & 8 & 3.7 & 38.994 & 32.374 & 0.204 \\
\hline
\end{tabular}

Table 1. The 15 seismic events of interest, where S > 0 (see Equation 2), in the period from 1955 to 1991 (data provided by the Portuguese National Laboratory for Civil Engineering and by the Portuguese Meteorological Institute). M, seismic magnitude; R, earthquake preparation radius; D, epicentre distance to the PG sensor; and S, ratio R D (see text).

Indeed, Dobrovolsky et al. [1979] developed the concept of the earthquake-preparation radius ( $\mathrm{R})$, which depends on the event magnitude $(\mathrm{M})$. This assumes the existence of an approximately circular region around the epicenter of an earthquake that undergoes elastic crustal deformation prior to the seismic events themselves. Dobrovolsky et al. [1979] estimated $\mathrm{R}$ as:

$$
R \approx 10^{0.43 M}
$$

Hence, taking into account the above arguments, it is likely that for relevant events (those that can actually influence the measurements), the PG sensor should be within a circle of radius $R$ (approximately). This means that the distance from the sensor to the event epicenter, $D$, must be smaller than $R$, i.e. $R \geq D$. This assumption enables the estimation of a dimensionless parameter:

$$
S=\frac{R}{D}-1
$$

which must be positive for seismic events of interest.

Applying this simple criterion in the analysis of the two seismic catalogs referred to above for the period of the sensor operation, from Sousa et al. [1992] and from the Portuguese Meteorological Institute database, there were 15 events that might be relevant. Table 1 gives some of the characteristics of these events, together with the corresponding $S$ values (see Equation 2). The seismic event in 1962 is not considered in this analysis as it corresponds to a year of high levels of artificial radioactivity, while the seismic event in 1975 was unfortunately missed because of the interruption of the dataset mentioned above.

Then, for the selected events we determined the Pearson's correlations of $S$ with the average values of the PG for three main periods: (i) 1 day before and 1 day after the events (PG_2); (ii) 7 days before and 7 days after the events (PG_14); and (iii) 14 days before and 14 days after the events (PG_28). Additionally, we considered the PG averages using only the midnight values, from 22:00 to 02:00 (UTC), as it is known from the literature that during this period the atmospheric activity is more stable [see Biagi et al. 2009]. In this way, three new cases were defined for the periods: 2 days (PG_2mn), 14 days (PG_14mn), and 28 days (PG_28mn). The results are presented and discussed in the next section.

\section{Results and discussion}

The analysis of the dependence of the different PG averages as a function of the dimensionless parameter $S$ (as discussed in section 3 ) is presented in Figure 3, and it tends to show negative associations between these two quantities (see Figure 4). Although the $p$ values for PG_14 and PG_14mn of 0.168 and 0.179 , respectively, do not allow these to be considered statistically significant, their correlation coefficients of -0.407 and -0.397 , respectively, do indicate this trend. These results highlight that the major influence of the seismic activity on the PG occurs over an interval of 14 days centered on the seismic event. Interestingly, this time interval of nearly two weeks around the earthquake occurrence is common to other seismic 


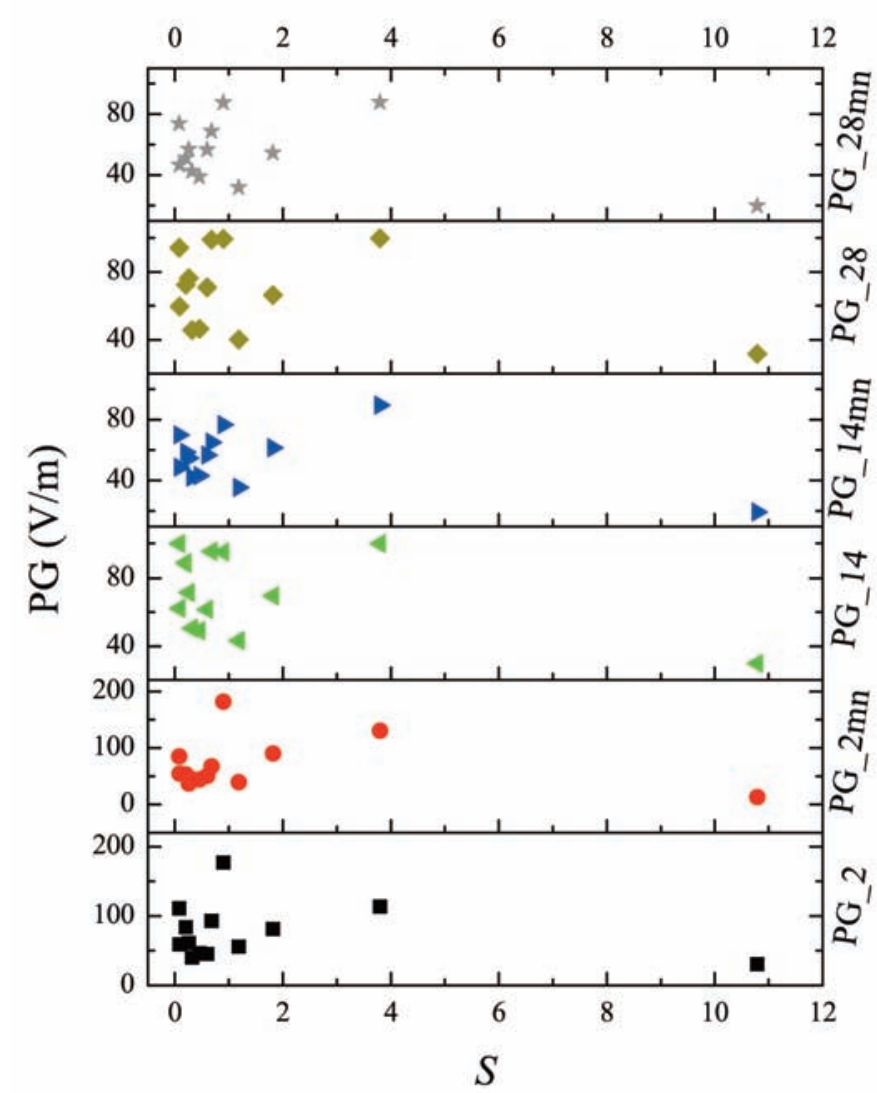

Figure 3. The PGs for the different averages considered in this study (PG_2, PG_2mn, PG_14, PG_14mn, PG_28, PG_28mn) as a function of S.

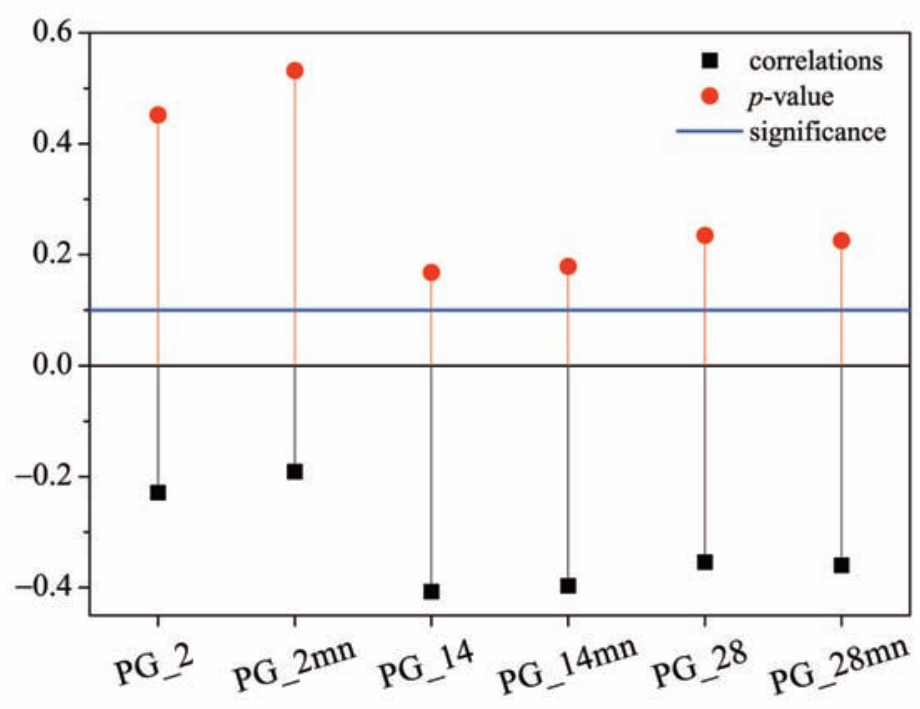

Figure 4. Statistics relating to the PG and S correlations.

precursors, like geomagnetic field anomalies [e.g. FraserSmith et al. 1990] and perturbations in very low frequency/low frequency radio transmissions [e.g. Biagi et al. 2009]. Moreover, similar intervals were also observed by Varostos et al. [1984] for the different phenomenon known as seismic electric signals. In addition, the analysis also shows that short-time averages, as with two days in this case, are insufficient to capture the effects of seismicity on the PG. This result might indicate that short-term variations in the PG caused by clouds, dust, or other phenomena, might overshadow the effects of seismic activity on the local electric environment.

This tendency to a negative association between PG and $S$ is also interesting. This reveals consistently decreasing PG values with $S$. This is physically expected, as can be seen when two events with the same magnitude but with the epicenter at different distances from the PG sensor are considered. Although both of these events will have similar tension fields during the earthquake preparation, it is expected that the nearest event (with the greater $S$ ) should create a greater tension field in the sensor region than the distant one (with the smaller $S$ ). For this reason, if radon emanation occurs in the zones where tensions are likely to alter the soil permeability, radon emanation due to the first of these events is expected to be higher in the zone of the sensor, as compared to that from the second event. This would naturally result in a more significant reduction in the PG associated with the first earthquake, with respect to the second earthquake.

\section{Final remarks}

To sum up, the present study has used simple statistical techniques to reveal indications that seismic activity leaves its mark on the atmospheric electrical field in a region close to an earthquake epicenter, with a trend to reduce the atmospheric electrical field.

This aspect opens the way to two main approaches. First, more profound examinations of the datasets are required. Special attention needs to be paid to the influence of meteorological variables on the PG, like relative humidity, precipitation, wind intensity and cloudiness, in line with other studies [e.g. Kachakhize et al. 2009], with the purpose of not restricting the data to fair-weather conditions. This also indicates that the present dataset can be complemented with measurements of the atmospheric radon levels during the period of operation of the PG sensor. This will provide a better understanding of the importance of radon in the lithosphere-atmosphere-ionosphere coupling. Secondly, the present method can be applied to PG datasets in other seismically active regions. Indeed, this last approach reveals the relevance of carrying out PG measurements worldwide, not only because of the great importance in itself [see Harrison 2005], but also because it might contribute in a significant way to the study of seismic precursors.

Acknowledgements. One of the authors (HGS) acknowledges the support of two Portuguese institutions: the Science and Technology Foundation (FCT) for the grant SFRH/BPD/63880/2009, and the Calouste Gulbenkian Foundation (FCG) for Estímulo à Criatividade e à Qualidade na Actividade de Investigação in the science program of 2010. We are grateful to the Portuguese Metereological Institute for allowing us to use their seismic database. Finally, important discussions of this study with Prof. Giles Harrison, Dr. Keri Nicoll, and Dr. Karen Aplin are strongly acknowledged. 


\section{References}

Biagi, P.F., L. Castellana, T. Maggipinto, D. Loiacono, L. Schiavulli, T. Ligonzo, M. Fiore, E. Suciu and A. Ermini (2009). A pre-seismic radio anomaly revealed in the area where the Abruzzo earthquake $(M=6.3)$ occurred on 6 April 2009, Nat. Hazard Earth Sys., 9, 1551-1556.

Borges, J., A.J.S. Fitas, M. Bezzeghoud and P. Teves-Costa (2001). Seismotectonics of Portugal and its adjacent Atlantic area, Tectonophysics, 337, 373-387.

Buforn, E., M. Bezzeghoud, A. Udias and C. Pro (2004). Seismic source in the Iberian-African plate boundary, Pure Appl. Geophys., 161, 623-646.

Dobrovolsky, I.P., S.I. Zubkov and V.I. Miachkin (1979). Estimation of the size of earthquake preparation zones, Pure Appl. Geophys., 117, 1025-1044.

Fraser-Smith, A.C., A. Bernardi, P.R. McGill, M.E. Ladd, R. A. Helliwell and O.G. Viilard (1990). Low-Frequency magnetic field measurements near the epicenter of the Ms 7.1 Loma Prieta earthquake, Geophys. Res. Lett., 17, 1465-1468.

Harrison, R.G. (2005). Columnar resistance changes in urban air, J. Atmos. Sol.-Terr. Phys., 67, 763-773.

Harrison, R.G., K.L. Aplin and M.J. Rycroft (2010). Atmospheric electricity coupling between earthquake regions and the ionosphere, J. Atmos. Sol.-Terr. Phys., 72, 376-381.

Kachakhidze, N. M. Kachakhidze, Z. Kereselidze and G. Ramishvili (2009). Specific variations of the atmospheric electric field potential gradient as a possible precursor of Caucasus earthquakes, Nat. Hazard Earth Sys., 9, 1221-1226.

Kachakhidze, M.K., Z.A. Kereselidze and N.K. Kachakhidze (2011). The model of self-generated seismo-electromagnetic oscillations of the LAI system, Solid Earth, 2, 17-23.

Liperovsky, V.A., C.-V. Meister, E.V. Liperovskaya, and V.V. Bogdanov (2008). On the generation of electric field and infrared radiation in aerosol clouds due to radon emanation in the atmosphere before earthquakes, Nat. Hazard Earth Sys., 8, 1199-1205.

Omori, Y., H. Nagahama, Y. Kawada, Y. Yasuoka, T. Ishikawa, S. Tokonami and M. Shinogi (2009). Pre-seismic alteration of atmospheric electrical conditions due to anomalous radon emanation, Phys. Chem. Earth, 34, 435-440.

Pierce, E.T. (1972). Radioactive fall-out and secular effects in atmospheric electricity, J. Geophys. Res., 77, 482-487.

Pierce, E.T. (1976). Atmospheric electricity and earthquake prediction, Geophys. Res. Lett., 3, 185-188.

Serrano, C., A.H. Reis, R. Rosa and P.S. Lucio (2006). Influences of cosmic radiation, artificial radioactivity and aerosol concentration upon the fair-weather atmospheric electric field in Lisbon (1955-1991), Atmos. Res., 81,
236-249.

Silva, H.G., M. Bezzeghoud, A.H. Reis, R.N. Rosa, M. Tlemçani, A.A. Araújo, C. Serrano, J.F. Borges, B. Caldeira and P.F. Biagi (2011). Atmospheric electrical field decrease during the $M=4.1$ Sousel earthquake (Portugal), Nat. Hazard Earth Sys., 11, 987-991.

Smirnov, S. (2008). Association of the negative anomalies of the quasistatic electric field in atmosphere with Kamchatka seismicity, Nat. Hazard Earth Sys., 8, 745749.

Sousa, M.L., A. Martins and C.S. Oliveira (1992). Compilação de catálogos sísmicos da região Ibérica. Relatório 36/92, NDA, Laboratório Nacional de Engenharia Civil, Lisboa.

Varotsos, P. and K. Alexopoulos (1984). Physical properties of the variations of the electric field of the earth preceding earthquake I, Tectonophysics, 110, 73-98.

\footnotetext{
*Corresponding author: Hugo Manuel Gonçalves da Silva, Universidade de Évora, Escola de Ciências e Tecnologia, Centro de Geofísica de Évora e Departamento de Física, Évora, Portugal email: hgsilva@uevora.pt.

(C) 2012 by the Istituto Nazionale di Geofisica e Vulcanologia. All rights reserved.
} 